\title{
Variables predictoras del acoso sexual en estudiantes universitarios
}

\section{Predictive variables of sexual harassment in university students}

\section{Referencia del artículo}

\author{
- Carmen María Véliz y Maribel Valenzuela \\ - Facultad de Humanidades \\ Universidad de San Carlos de Guatemala \\ Correo electrónico: carmencita.veliz@gmail.com \\ https://orcid.org/0000-0003-2080-5757
}

Véliz, C. y Valenzuela, M. (2020). Variables predictoras de acoso sexual en estudiantes universitarios. Revista Guatemalteca de Educación Superior, 3(1), 32-46.

DOI: https://doi.org/10.46954/revistages.v1i1.3

$\begin{array}{ll}\text { Recibido } & 02 / 05 / 2020 . \\ \text { Revisado } & \text { 15/05/2020. } \\ \text { Aceptado } & \text { 09/06/2020. }\end{array}$

\section{Resumen}

El objetivo es presentar resultados del trabajo de investigación sobre el acoso sexual en relación con las variables predictoras en el espacio universitario, para convertirse en un marco de referencia para la comunidad educativa. Este problema es de alta incidencia en el contexto guatemalteco. El estudio de corte transversal se realizó con 210 estudiantes de dos unidades académicas de la USAC, se realizó un muestreo probabilístico por racimos de una población, de un estimado de 420 estudiantes. La investigación es cuantitativa, no experimental y explicativa; se utilizó la técnica del cuestionario. Las variables del estudio fueron acoso sexual, sexismo ambivalente, autoestima y rendimiento académico. El $74.3 \%$ de los estudiantes que contestaron el cuestionario, afirman haber sufrido alguna forma de acoso sexual en el espacio universitario, de este porcentaje el $68.4 \%$ reportaron que su acosador era otro estudiante, y un $88.7 \%$ que su acosador fue un hombre. Se 
generó un modelo con un adecuado ajuste de acuerdo a la prueba Hosmer-Lemeshow, $\mathrm{p}>0.05$, en el que aportaron significativamente dos variables, el ser mujer que aumenta un 3.43 la probabilidad de sufrir acoso sexual, y tener bajos niveles de autoestima social que aumenta un 1.5 dicha probabilidad. El acoso sexual puede predecirse por variables presentes en la víctima como ser mujer y tener baja autoestima social, sin embargo, las causas que explican el problema se relacionan más con aspectos sociales y culturales como el machismo, por lo que el problema debe de abordarse a este nivel.

\section{Abstract}

The objective of this research is to present the results of the study work on sexual harassment in relation to the predictive variables in the university space to become a frame of reference for the educational community. This problem is of high incidence in the Guatemalan context. The cross-sectional study was carried out with 210 students from two academic units of the USAC, a probabilistic sampling was carried out by clusters of a population of an estimated 420 students. The research is a quantitative one, non-experimental and explanatory, the questionnaire was used. The variables of the study were sexual harassment, ambivalent sexism, self-esteem and academic performance. $74.3 \%$ of the students who took the questionnaire reported that they suffered some form of sexual harassment in the university space, of this percentage $68.4 \%$ reported that their harasser was another student, and $88.7 \%$, their harasser was a man. A model with an adequate adjustment was generated according to the Hosmer-Leme show test, $p>0.05$, in which two variables contributed significantly, being a woman that increases the probability of sexual harassment by 3.43 , and having low levels of social self-esteem. which increases the probability by 1.5. Sexual harassment can be predicted by variables present in the victim such as being a woman and having low social self-esteem, however, the causes that explain the problem are more related to social and cultural aspects such as machismo, so the problem must be addressed at this level.
Palabras clave

violencia de género, cultura

machista, autoestima, psicoeducación, ruta de denuncia.

\section{Keywords}

gender violence, macho culture, self-esteem, psychoeducation, complaint route 


\section{Introducción}

El acoso es un problema que históricamente ha sido objeto de estudio y discusión por su alta prevalencia y normalización. Este se entiende en el presente estudio como el "comportamiento en función del sexo, de carácter desagradable y ofensivo para la persona que lo sufre. Para que se trate de acoso sexual es necesaria la confluencia de ambos aspectos negativos: no deseado y ofensivo" (Organización Internacional del Trabajo, 2013). Fue a partir de la promulgación de la Convención Interamericana para Prevenir, Sancionar y Erradicar la Violencia Contra la Mujer en 1994, que se reconocieron las distintas formas de violencia que afectan a las personas, específicamente a las mujeres, como resultado de un sistema patriarcal que favorece las relaciones de poder; esto produjo un incremento en el total de denuncias sobre este tipo de violencia (Logroño, 2009). En Guatemala el acoso sexual no se encuentra tipificado como un delito en el código penal, y es por ello que solo se puede llegar, en algunos casos, a una conciliación con el agresor, lo que genera una persistente impunidad (Organización Internacional del Trabajo, 2013). Con respecto al espacio universitario, en un estudio exploratorio sobre el acoso sexual realizado en la Universidad de San Carlos de Guatemala se encontró que 9 de cada 10 estudiantes se ven afectados por el acoso sexual, y la mayoría de los acosadores conocidos son catedráticos (Asociación de Estudiantes Universitarios, 2019).

Estos datos evidencian la gravedad del problema cuyas explicaciones resultan complejas. Tradicionalmente, algunos autores afirman que la educación superior no fue un espacio en el que cupieran las mujeres, ya que era privilegiado para los hombres, algo que fortalecía la división sexual del trabajo y de este modo, eran quienes tenían la oportunidad de desarrollo académico y profesional (Papadópulos \& Radakovich, 2004). Y si bien en la actualidad estos escenarios han cambiado, la realidad que viven las mujeres en la universidad es difícil (Ordorika, 2015).

El acoso sexual, interpretado como violencia de género, es una forma de violencia aceptada en el espacio universitario bajo una tolerancia silenciosa, en donde muchos conocen el problema, pero pocos actúan para solucionarlo y además, se promueve una 
cultura de silencio y de no denuncia (Barreto, 2017). La misma autora afirma que es vital que los delitos sobre violencia sexual sean reconocidos y sancionados por las autoridades para genera un cambio de cultura.

Es así como el acoso sexual forma parte de una cultura institucional de género, en donde se promulga el comportamiento masculino que niega el valor de las mujeres en el mundo (Mingo \& Moreno, 2015), valiéndose de relaciones de poder. A partir de esta conceptualización, el acoso sexual se describe como una manifestación altamente violenta de la discriminación basada en género, respaldada por pautas sociales y culturales.

En este punto surge uno de los aspectos más preocupantes del acoso sexual: la normalización de la problemática. Frecuentemente se culpa a la víctima de acoso sexual casi de manera natural, y esto se explica por el condicionamiento social legitimador que aprueba esta forma de violencia (Castaño-Castrillón et al., 2010). La persona que es víctima llega incluso a ser criminalizada (Echeverría, Paredes, Diódora, Batún, \& Carrillo, 2017), y con esto queda más vulnerable.

Resulta conveniente profundizar en el acoso sexual desde un enfoque explicativo, para determinar aquellas variables que podrían aumentar la probabilidad de que una persona sea víctima de acoso sexual. A su vez, la teoría que se genera permitirá que se planteen y ejecuten acciones estratégicas para disminuir la incidencia e impacto del acoso sexual en la universidad. Con base a esta descripción del problema del acoso sexual se estableció como objetivo general: interpretar el acoso sexual en relación con las variables predictoras en el espacio universitario para convertirse en un marco de referencia para la comunidad educativa. Para alcanzarlo se plantearon los siguientes objetivos específicos: determinar las variables predictivas del acoso sexual, para caracterizar el fenómeno en el espacio universitario; analizar las experiencias de acoso sexual desde las voces de los estudiantes para evidenciar las causas del fenómeno. 
Facultad de Humanidades

Escuela de Estudios de Postgrado

\section{Materiales y Métodos}

La población se constituyó de estudiantes de último año/semestre que estuvieran inscritos, ya sea en la Facultad de Veterinaria o en la Escuela de Ciencias Psicológicas de la Universidad de San Carlos de Guatemala, considerando que los estudiantes en esta fase de su vida universitaria han podido tener más y variadas experiencias. La población aproximada correspondió a 420 estudiantes.

Se utilizó un muestreo probabilístico por racimos, también llamados clusters o conglomerados. Los clusters fueron todas las sesiones de último año de la carrera universitaria de ambas unidades académicas, y se tomaron en cuenta todos los electivos y jornadas de las mismas, sumando un total de ocho clusters. Se hizo una selección aleatoria de cuatro, utilizando el software SurveyMonkey, con un nivel de confianza de $95 \%$ y un margen de error de 5\%. En los clusters seleccionados se evaluó el total de los sujetos del estudio, o en otras palabras, todos los estudiantes que se encontraran presentes en los momentos de aplicación de instrumentos, sumando un total de 210 estudiantes.

Se tomaron en cuenta como criterios de inclusión: ser estudiante de una de las dos unidades académicas seleccionadas (sin importar edad y sexo), estar cursando al menos un curso del último año/ semestre de la carrera, estar inscrito en el ciclo 2020 de la Universidad de San Carlos y firmar el consentimiento informado. Los criterios de exclusión fueron rellenar de forma aleatoria los instrumentos, no rellenar la totalidad de los instrumentos y presentar algún tipo de discapacidad auditiva, visual o mental.

El alcance de la investigación fue explicativo, se utilizó un enfoque cuantitativo y un diseño no experimental y transversal, en el que se aplicaron cuestionarios y escalas estandarizadas. Para la recolección de datos se utilizaron fuentes primarias. Los instrumentos se aplicaron durante el horario de clases de los estudiantes, con las previas autorizaciones correspondientes. La aplicación duró aproximadamente media hora por cluster, y se solicitó el apoyo del catedrático encargado del salón. 
Como parte de las consideraciones éticas de la investigación, se solicitó el consentimiento informado de los participantes antes de la aplicación de instrumentos. Se respetó la voluntariedad a querer o no formar parte del estudio. Asimismo, los datos obtenidos fueron debidamente resguardados para mantener la confidencialidad y anonimato de los participantes, sin excepción.

Las variables del estudio fueron autoestima, sexismo ambivalente, rendimiento académico y otras variables determinadas en la encuesta sociodemográfica. Para medirlas se aplicaron los siguientes instrumentos: encuesta sociodemográfica, Cuestionario para la medición del acoso sexual en instituciones de educación superior (IES) de Lourdes Larrea, Inventario de sexismo ambivalente (ASI) de Glick y Fiske, y Escala de autoestima de Coopersmith (SEI) versión de adultos.

Para el análisis de datos se inició con la estadística descriptiva para reportar características demográficas de la población. Asimismo se hicieron estimaciones para las pruebas de hipótesis con un 95\% de confianza. Para cada instrumento aplicado se realizó análisis de consistencia interna usando el alfa de Cronbach >.6. Finamente se realizó análisis de regresión logística binomial, evaluando los factores protectores y de riesgo para el acoso sexual.

Para llevar a cabo este análisis estadístico se estimaron los odds ratio (OR) sin ajustar como medida de asociación entre cada variable expuesta. Se generó un modelo predictivo en el que se incluyeron las variables que mostraron un valor $\mathrm{p} \leq .1$ (índice de OR que indica probabilidad). Se hicieron todas las pruebas del modelo final con un nivel de confianza $\alpha=.05$. Todos los análisis se realizaron en el programa IBM SPSS Statistics 25.

\section{Resultados}

\section{Caracterización de la muestra de estudio}

Para caracterizar la muestra de estudio se aplicó una encuesta sociodemográfica, los principales datos que se obtuvieron se presentan a continuación. La mayor parte de la muestra la 
representaron mujeres, siendo un $77.1 \%$ de total, lo cual tiene que ver con las unidades académicas que formaron parte del estudio, siendo psicología una unidad en la que estudian mayoritariamente mujeres, y veterinaria una unidad en donde es relativamente igual la cantidad de mujeres y hombres. El $58.1 \%$ de la muestra correspondió a estudiantes de psicología y el $41.9 \%$ a estudiantes de veterinaria.

La edad de la muestra se encuentra entre 21 a 25 años en su mayoría, representando un $66.7 \%$ del total. Con respecto a su ocupación, un $58.6 \%$ comenta que únicamente estudia, y un $41.4 \%$ que estudia y trabaja. En cuanto a sus vínculos familiares, un $90 \%$ de la muestra indica no tener hijos. Un 69.5\% afirma no tener pareja sentimental actualmente. El $88.6 \%$ tiene hermanos, entre uno y cuatro en su mayoría, solo el $13.4 \%$ afirma tener más de cuatro hermanos. Únicamente el 5.2\% refieren vivir solos actualmente. La gran mayoría de la muestra, representando un $76.7 \%$, califica sus relaciones sociales en general como satisfactorias.

Con respecto a su rendimiento académico, únicamente el $7.1 \%$ de la muestra indica tener un promedio menor a 69 puntos, y comentan no haber tenido una disminución del mismo en el último año, a pesar de que la mayor parte de la muestra comenta haber sido víctima de acoso sexual en este mismo año. Estos resultados más el análisis de estadística inferencial, denotan que no existe relación entre el haber sido víctima de acoso sexual y la disminución del rendimiento académico.

\section{Pruebas de normalidad}

Como se mencionó anteriormente, se aplicaron tres instrumentos estandarizados: Inventario de sexismo ambivalente (ASI), Escala de autoestima (SEI) versión de adultos y Cuestionario para la medición del acoso sexual en instituciones de educación superior (IES). Con los dos primeros se realizaron pruebas de normalidad y medidas de tendencia central, con el último no, ya que a través del mismo únicamente se identificó la presencia-ausencia de acoso sexual y algunas características sobre las experiencias. 
Tabla 1. Medidas de tendencia central del sexismo ambivalente

\begin{tabular}{lll}
\hline & $\begin{array}{l}\text { Inventario de sexismo } \\
\text { ambivalente }\end{array}$ & Escala de autoestima \\
\hline Media & 57 & 16.3158 \\
\hline Desviación estándar & 19.053 & 4.921 \\
\hline Mínimo & 22 & 0 \\
\hline Máximo & 111 & 25 \\
\hline
\end{tabular}

Fuente: Véliz (2020).

En primer lugar, las medidas de tendencia central del Inventario de sexismo ambivalente (ASI) (ver tabla 1) denotan que no hay valores nulos de sexismo ambivalente, es decir, aunque sea bajo, todos los sujetos entrevistados presentan indicadores de sexismo ambivalente. Ahora bien, hay que considerar que la desviación estándar del instrumento es muy amplia ( $\sigma=19.05)$, lo que implica que los datos están muy dispersos y se debe ser cauteloso al momento de realizar generalizaciones con este instrumento.

Para medir la confiabilidad de los instrumentos se calculó el alfa de Cronbach ( $\alpha$ ). El Inventario de sexismo ambivalente (ASI) resultó ser un instrumento confiable en el estudio, teniendo un valor $\alpha=.886$ en la escala general. En la subescala de sexismo ambivalente benévolo se obtuvo un $\alpha=.765$ y en la subescala de sexismo ambivalente hostil, un $\alpha=.882$, la primera cuenta con valores aceptables de confiabilidad y la segunda con valores buenos. Esto quiere decir que es un instrumento que produce resultados consistentes y coherentes, dando solidez al estudio.

Con respecto a la Escala de autoestima de Coopersmith (SEI) versión de adultos, las medidas de tendencia central muestran que la media está sobre el valor central del instrumento (ver tabla 1), lo cual implica que los sujetos del estudio tienen niveles de autoestima medios o altos en su mayoría. La desviación estándar es media $(\sigma=4.921)$ por lo que, aunque no están tan dispersos los datos, el instrumento debe analizarse con cautela. Asimismo, el instrumento obtuvo un valor de $\alpha=.827$, lo que quiere decir que tiene niveles buenos de confiabilidad. Con estos datos se puede asumir que el instrumento, en términos general, es bueno. 
Facultad de Humanidades

Escuela de Estudios de Postgrado

\section{Variables predictoras del acoso sexual}

Para determinar las variables predictoras del acoso sexual se realizó un análisis de regresión logística binomial. La interacción de las variables dentro del modelo permitió obtener la siguiente información: el modelo presentó un adecuado ajuste de acuerdo a la prueba de Hosmer-Lemeshow, X2 = 8.29-, $p>0.05$, y explicó el $23 \%$ de la varianza (Nagalkerke R2). Asimismo, se clasificó correctamente el $77 \%$ de los casos.

A pesar de esta buena interacción en el modelo, las únicas variables que aportaron información significativa para predecir el acoso sexual fueron: el sexo y la autoestima social. Las mujeres presentaron 3.43 más probabilidades de reportar haber sufrido acoso sexual. Por otro lado, un aumento en el punteo de autoestima social se asoció con una reducción de las probabilidades de reportar experiencias de acoso sexual por 1.5 veces.

Es necesario enfatizar que dichas variables que resultaron predictoras en el presente estudio no son causas determinantes del acoso sexual, ya que ello implicaría culpar a la víctima. Lo que pretendió el análisis fue establecer las características de la víctima que aumentan la probabilidad de sufrir acoso sexual, sin ser estas en sí mismas las causas finales. El acoso sexual se explica mejor con aspectos sociales y culturales que con las personas a nivel individual. Bien explica Butler (2007), los comportamientos que se tienden a caracterizar como violencia de género, entre ellos el acoso sexual, se convierten en un efecto cultural, que surge de la repetición de convencionalismos de manera ritualizada.

\section{Experiencias de acoso sexual desde las voces de los estudiantes}

Se consideró importante describir las experiencias de acoso sexual que viven los estudiantes. 
Figura 1. Presencia de acoso sexual en la muestra de estudio

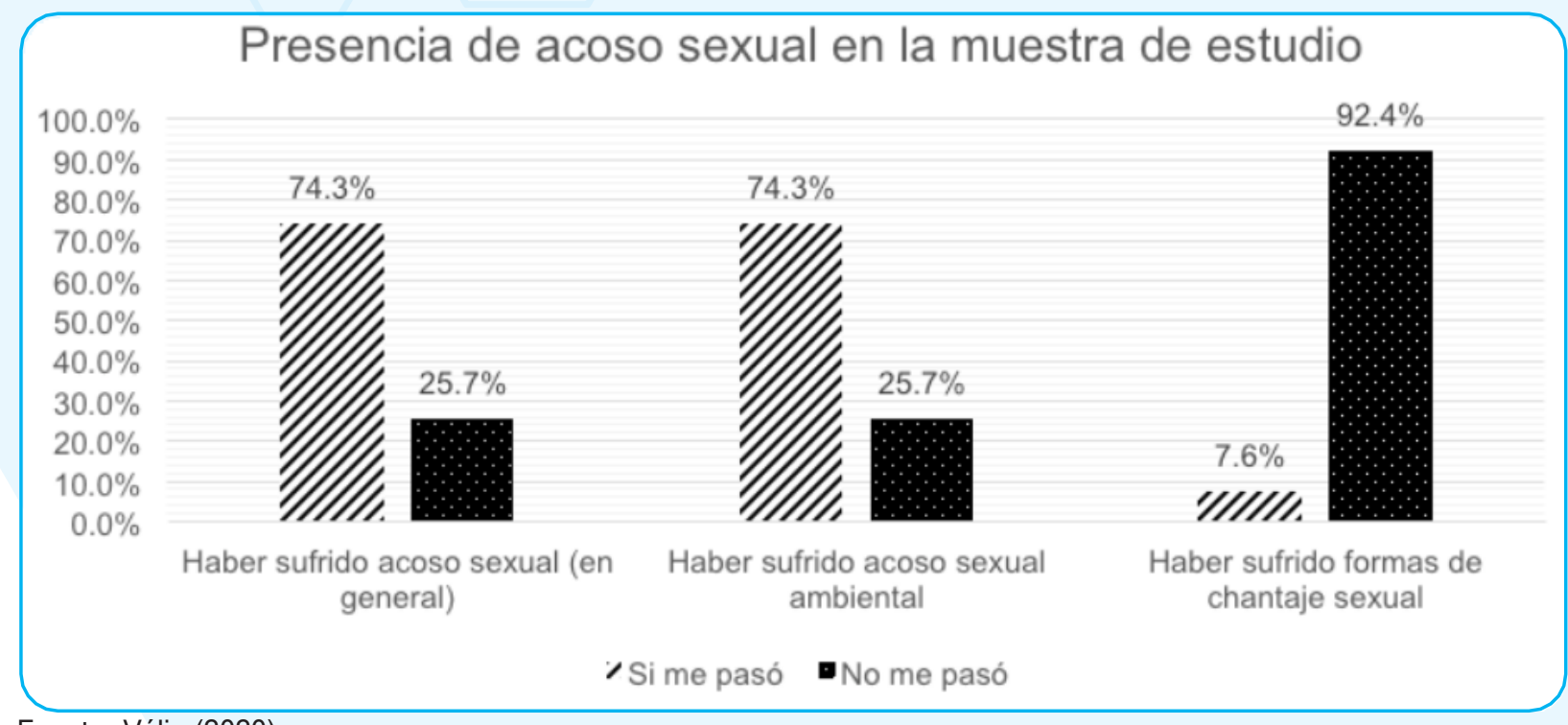

Fuente: Véliz (2020).

Se logró identificar que el $74.3 \%$ de la muestra refiere haber sufrido alguna forma de acoso sexual en el espacio universitario. La forma de acoso sexual que prevalece es el acoso sexual ambiental, el cual se da cuando existe un ambiente hostil, lo suficientemente intenso para generar un clima abusivo, humillante o amenazador para la víctima como lo describe (Larrea, 2018). El chantaje sexual no fue tan prevalente en el estudio.

Las personas que tienden a acosar en un mayor nivel son los propios estudiantes, es decir, compañeros de estudio, representando un $68.40 \%$ de las personas que acosan. El segundo lugar lo ocupan los docentes universitarios con un $25.28 \%$. Asimismo, un $88.7 \%$ de los acosadores son hombres.

\section{Propuesta para minimizar la incidencia e impacto del acoso sexual}

Los resultados del estudio permitieron visibilizar la alta incidencia, características y variables predictoras del acoso sexual en el espacio universitario, lo cual permitió construir una propuesta para minimizar el impacto de esta problemática. Se consideraron elementos en la propuesta realizada por García (2016), tomando en cuenta los resultados del presente estudio y las necesidades evidenciadas. 
Facultad de Humanidades

Escuela de Estudios de Postgrado
Se planteó como objetivo de la propuesta: Generar condiciones de prevención y protección hacia estudiantes que han sido y/o pueden ser víctimas de acoso sexual en la Universidad de San Carlos de Guatemala. Para lograrlo se establecieron tres acciones estratégicas, las cuales se integran en una metodología participativa, que se sugiere puede ser desde el diseño de investigación-acción, tomando como punto de partida el presente estudio.

Las acciones estratégicas planteadas son:

- Campaña de comunicación: consiste en dos acciones operativas, la difusión y socialización de los resultados de la presente investigación, elementos publicitarios. Se recomienda crear un mensaje principal tipo eslogan y basarse en una línea gráfica. El objetivo de la campaña es generar y transmitir el mensaje de cero tolerancia al acoso sexual en el espacio universitario.

- Programa de psicoeducación: para esta acción se propone crear planificaciones de talleres por medio de los cuales se alcance el objetivo de informar y generar una cultura de respeto y cero tolerancia al acoso sexual en el espacio universitario. Para ello se proponen dos modalidades, la primera son talleres contra la violencia de género dirigidos a toda la población universitaria; y la segunda son talleres de empoderamiento dirigidos a las mujeres. La metodología a utilizar será la psicoeducación.

- Ruta de denuncia: finalmente se considera vital en la propuesta, coordinar con las autoridades administrativas de las unidades académicas la construcción de una ruta de denuncia que permita la activación de protocolos de manera rápida y eficiente al momento de identificar un posible caso de acoso sexual en el espacio universitario.

La propuesta completa se encuentra en el informe de tesis realizada en la Facultad de Humanidades. Asimismo, es importante aclarar que, si bien esta sugerencia de trabajo no puede erradicar un problema tan complejo en su totalidad, si puede incidir en disminuir la probabilidad que ocurra, así como en el impacto negativo que genera en la cultura institucional de la universidad. 


\section{Discusión}

Con base a los resultados obtenidos y contrastándolos con estudios previos, el machismo construye la cultura institucional de la universidad, lo que conduce a una buena parte de los hombres a expresar hostilidad de manera abierta y sistemática contra las mujeres (Mingo, 2016). Esto implica que todas las variables que podrían llegar a ser predictoras del acoso sexual, son filtradas y delimitadas por una cultura machista que condena lo femenino, enaltece la masculinidad hegemónica y circunscribe las relaciones a ciertos patrones heteronormativos.

Es por ello que ser mujer, tener bajos niveles de autoestima social y algunas otras actitudes relacionadas con las interacciones sociales resultaran predictoras del acoso sexual en el estudio. No se trata de que estas variables en sí mismas sean las causas, es la cultura machista la que determina que elementos van a permitir, facilitar y regularizar el acoso sexual, como una acción legitimada social y culturalmente.

Muchas de las actitudes o variables que tienden a determinar a una persona más vulnerable ante el acoso sexual, son aspectos bastante comunes y naturales, pero al venir de una mujer pueden ser interpretados como permisos u ofrecimientos de índole sexual. No es que estas actitudes sean predictoras del acoso sexual en sí mismas, pero dentro del contexto machista forman parte de la performatividad de género, en donde por medio del discurso y el lenguaje se genera una relación causal entre dichas actitudes y el ser víctima de acoso sexual (Butler, 2007).

En diversos estudios se confirma que la mayoría de víctimas de acoso sexual tienden a ser mujeres, quienes por razones de género, sufren una serie de conductas violentadas basadas principalmente en prejuicios y estereotipos (Aguilar, Alonso, Melgar, \& Molina, 2009). Sin embargo, los hombres también sufren de acoso sexual y de acuerdo a estudios, pueden llegar a ser violentados, acosados y hostigados; en ambos casos, la cultura de denuncia es una meta común a alcanzar (Echeverría et al., 2017). 
Facultad de Humanidades

Escuela de Estudios de Postgrado
En esta línea es importante resaltar las características de las personas que acosan, pues generalmente en discursos coloquiales se escucha la frase a "los hombres también los acosas", y como se menciona, efectivamente algunos hombres viven experiencias de acosos sexual en el espacio universitario, destacando que siempre en una menor proporción que las mujeres. Sin embargo, como se pudo ver en los resultados del estudio, la gran mayoría de los acosadores son hombres.

En otras palabras, si un hombre también puede ser víctima de acoso sexual, la persona que los acosa tiende a ser otro hombre, lo cual remarca que el tema sigue siendo de índole de género y del permiso cultural que tiene lo masculino hegemónico de tener este tipo de conductas violentas y dominantes en la universidad (Mingo, 2016). Esto se relaciona directamente con la negación simbólica de la mujer y todo lo que represente el elemento femenino en los espacios sociales, en este caso la universidad, lo cual se valida por medio de expresiones lingüísticas-semióticas (Butler, 2007).

Hay que mencionar que generalmente la persona o personas que exponen a estudiantes a diferentes formas de acoso sexual poseen una posición jerárquica en la comunidad universitaria, determinada por relaciones de poder sobre la persona que agreden o acosan. Es así como el acoso sexual es un problema producto de diferencias de poder establecidas, validadas y legitimadas por una cultura institucional (Cuenca, 2015)

Finalmente vale la pena mencionar que, a partir de este tipo de discusiones, se han creado campañas en contra de la violencia de género como respuesta al acoso sexual que constantemente sufren estudiantes en el espacio universitario, en especial las mujeres (Asociación de Estudiantes Universitarios, 2019). En la misma Universidad de San Carlos de Guatemala se ha consolidado el Instituto de la Mujer, fundado formalmente en el 2002, cuyo objetivo es propiciar la prevención, sensibilización, investigación y erradicación de todas las manifestaciones que llevan a la violencia sexual. Entre estas, el acoso sexual en todas sus formas (García, 2016), por lo que los esfuerzos para generar este tipo de propuestas deben ser impulsados y fortalecidos. 


\section{Agradecimientos}

Se agradece a las autoridades y docentes de la Escuela de Ciencias Psicológicas y de la Facultad de Veterinaria y Zootecnia, por su apoyo y apertura para la realización de la presente investigación. Asimismo a los estudiantes que participaron, por su disposición, colaboración y confianza en el presente estudio sobre acoso sexual con sus experiencias y creencias, en un esfuerzo académico para visibilizar el problema.

\section{Referencias}

Aguilar, C., Alonso, M., Melgar, P., \& Molina, S. (2009). Violencia de género en el ámbito universitario. Medidas para su superación. Revista Interuniversitaria de Pedagogía Social, (16), 85-94. https://doi.org/10.7179/PSRI_2009.16.06

Asociación de Estudiantes Universitarios. (2019). Estudio exploratorio sobre el acoso sexual en la Universidad de San Carlos de Guatemala.

Barreto, M. (2017). Violencia de género y denuncia pública en la universidad. Revista Mexicana de Sociología, 79(2), 261-286.

Butler, J. (2007). El género en disputa. El feminismo y la subversión de la identidad. (Paidós Ibérica S.A., Ed.). Barcelona.

Castaño-Castrillón, J. J., González, E. K., Guzmán, J., Montoya, J., Murillo, J., Páez-Cala, M., ... Velásquez, Y. (2010). Acoso sexual en la comunidad estudiantil de la comunidad de Manizales (Colombia). Estudio de corte transversal. Revista Colombiana de Obstetricia y Ginecología, 61(1), 18-27. https://doi. org/10.18597/rcog.303

Cuenca, C. (2015). Factores precipitantes del acoso sexual laboral en España. Revista Mexicana de Sociología, 77(4), 525-554.

Echeverría, R., Paredes, L., Diódora, M., Batún, L., \& Carrillo, C. (2017). Acoso y hostigamiento sexual en estudiantes universitarios: Un acercamiento cuantitativo. Enseñanza e Investigación En Psicología, 22(1), 15-26.

García, A. (2016). Campaña Educativa de información del acoso sexual en el campus central de la Universidad de San Carlos de Guatemala. 


\section{Sobre la autora}

Larrea, M. de L. (2018). ¿Cómo se mide el acoso sexual? Sistematización de la experiencia de construcción de contenidos de un instrumento para la medición del acoso sexual en instituciones de educación superior del Ecuador. Quito.

Logroño, J. (2009). Situación de acoso, abuso y otros delitos sexuales en el ámbito de la educación superior. Caso Universidad Central de Ecuador. Paraguay.

Mingo, A. (2016). «iPasen a borrar el pizarrón!» Mujeres en la universidad. Revista de La Educación Superior, 45(178), 1-15. https://doi.org/10.1016/j.resu.2016.03.001

Mingo, A., \& Moreno, H. (2015). El ocioso intento de tapar el sol con un dedo: violencia de género en la universidad. Perfiles Educativos, 37(148), 138-155. https://doi.org/10.22201/ iisue.24486167e.2015.148.49318

Ordorika, I. (2015). Equidad de género en la Educación Superior. Revista de La Educación Superior, 44(174), 7-17. https://doi. org/10.1016/j.resu.2015.06.001

Organización Internacional del Trabajo. (2013). El hostigamiento o acoso sexual.

Papadópulos, J., \& Radakovich, R. (2004). Educación Superior y Género en América Latina y el Caribe. Informe Sobre La Educación Superior En América Latina y El Caribe 2000-2005, 117-128.

Carmen María Véliz Escobar es licenciada en psicología, especializada en psicología clínica comunitaria y con maestría en docencia universitaria. Ha participado en diversas investigaciones en la UIP-Usac y como consultora independiente. Actualmente trabaja en proyectos de protección infantil y prevención de la violencia en NNAJ.

Copyright (c) Carmen María Véliz y Maribel Valenzuela

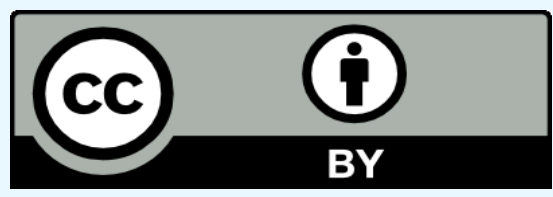

Este texto está protegido por una licencia CreativeCommons 4.0.

Usted es libre para compartir, copiar y redistribuir el material en cualquier medio o formato y adaptar el documento, remezclar, transformar y crear a partir del material para cualquier propósito, incluso comercialmente, siempre que cumpla la condición de atribución: usted debe reconocer el crédito de una obra de manera adecuada, proporcionar un enlace a la licencia, e indicar si se han realizado cambios. Puede hacerlo en cualquier forma razonable, pero no de forma tal que sugiera que tiene el apoyo del licenciante o lo recibe por el uso que hace. 